\title{
Presentation of pulmonary tuberculosis with or without co-existing type 2 diabetes mellitus - a prospective study
}

\section{ABSTRACT}

Background. Many studies depict that pulmonary tuberculosis in a patient with type 2 diabetes mellitus (T2DM) have some different and specific presentations. Aims and objective. Present study was performed to observe the various presentations in a patient with tuberculosis and T2DM.

Materials and methods. The study was performed on 105 patients with and without T2DM and sputum positive for tuberculosis divided into tuberculosis and diabetes mellitus (TBDM) group (55 patients) and tuberculosis without T2DM (TB) group (50 patients). Results were analyzed using detailed clinical history, systemic examination, radiological examination (classified in eleven patterns) and sputum smear examination (grading was done).

Results. The mean age of TBDM group was $51.2 \pm 8.05$ and the group was older than patients in TB group where mean age was $39.5 \pm 9.2$ years. Duration of T2DM ranged from 6 month to 10 years, with mean $4.21 \pm 1.86$ years. Hemoptysis was present in $40 \%$ of patients of TBDM group. Six (12\%) in TB group and 23 $(41.8 \%)$ patients in TBDM group were having $3+$ grade sputum smear positivity.

Conclusion. Significant difference was found in presentations in patients of TB with T2DM as compared to patients without T2DM. (Clin Diabetol 2016; 5, 5: 159-163)

Key words: T2DM, tuberculosis, sputum smear, pulmonary tuberculosis

Address for correspondence:

Dr Rakesh Kumar Shahi

I-83 Rapti Nagar Phase-IV Near BPCl, Gorakhpur

Uttar Pradesh, 273001

e-mail: Drrkshahigkp@gmail.com

Clinical Diabetology 2016, 5, 5, 159-163

DOI: $10.5603 /$ DK. 2016.0028

Received: 29.02.2016

\section{Introduction}

There are around 2 billion people who are infected with Mycobacterium tuberculosis; out of that around 11 million have active tuberculosis. The yearly incidence of tuberculosis cases and number of death are raising in developed and developing countries [1]. The high rise in the incidence of death due to Mycobacterium tuberculosis infection in middle and low income countries are due to ageing of their population and changing lifestyles and diets [1].

Out of total active cases of tuberculosis in world, $85 \%$ burden of TB cases is found in poor countries and of this, India bears $50 \%$ of the cases. According to World Health Organization (WHO), around 1.5 million people die due to $M$. tuberculosis infection every year [2].

As per International Diabetes Federation Atlas which was released in 2015, a total of 415 million people are living with diabetes, which is expected to rise to 642 million by 2040 [3].

T2DM has been reported to be independently associated with an unfavorable outcome of pulmonary TB [1]. T2DM patients with TB have been reported to have high rate of cavitary lesions, less sputum positivity and a paucity of symptoms and signs as compared to patients with TB without T2DM [4]. T2DM is found to be a risk factor for TB related death; one study reported two fold higher risk of death due to diabetes in tuberculosis comparing to patients without T2DM [1].

Present study was performed to analyze various clinical and radiological presentations in patients with pulmonary tuberculosis and T2DM.

\section{Materials and methods}

A prospective study was performed on confirmed 105 pulmonary tuberculosis patients admitted to the Department of Tuberculosis and Respiratory Diseases of 
BRD Medical College and Nehru Chikitsalya Gorakhpur from January 2013 to September 2015.

A written Informed consent from all patients and Institutional Ethics Committee approval was obtained before starting the study.

Patient with sputum positive for TB of both genders registered between $1^{\text {st }}$ January 2013 and $31^{\text {st }}$ September 2015 were included in present study. Patients with known HIV-positive status and with impaired glucose test or impaired fasting glucose on OGTT were excluded from the study. Also patients with other immune-compromised state, sputum negative patients and patients with infection were excluded from the present study.

The participants were recruited either while they were on treatment for TB or diagnosed as having TB.

Out of 105 patients, 55 (52.38\%) were having T2DM (TBDM) whereas 50 (47.62\%) patients presented with normal glucose tolerance (TB) which served as control group.

All patients were subjected to detailed clinical history, systemic examination, radiological examination, sputum smear examination for acid fast bacilli staining (fluorescent staining), blood glucose (fasting and postprandial) and glycated hemoglobin $\left(\mathrm{HbA}_{1 \mathrm{c}}\right)$. Patients were also investigated for complete blood count, serum creatinine, serum urea, serum bilirubine, alanine aminotransferase, aspartate aminotransferase, alkaline phosphatase and referred to Integrated Counseling and Testing Centres (ICTC) to know HIV infection status.

Patients were advised to collect two sputum samples in sterile sputum vial. Using the Revised National Tuberculosis Control Programme (RNTCP) guidelines in our hospital laboratory, patient received sputum containers with instructions to provide sputum specimens, which are then subjected for sputum examination.

Two sputum specimens were collected over one, or two consecutive days. Of the two sputum specimens, one was collected on the spot and the other was an early morning specimen, collected at home by the patient.

At laboratory, the sputum sample was processed by fluorescent microscopy, in which slides stain with auramine (Fluorochrome) rhodamine stain. In a well stained auramine smear acid-fast bacilli (AFB) appear as yellowish fluorescent bacilli against a dark background. Slides remained in contact with auramine for at least 15 minutes and not more than 30 minutes. Complete distaining was done with $0.5 \%$ acid alcohol and counterstained with $5 \%$ potassium permanganate.

Grading of the smears was done as per the International Union Against Tuberculosis and Lung Disease (IUATLD)/WHO scale as negative (zero AFB/1 length), scanty (1-29 AFB/1 length), 1+ (30-299 AFB/1 length),
$2+(10-100$ AFB/1 field on average) and $3+(>100$ AFB/1 field on average) at 200-250 $\times$ magnification (30 fields $=300$ high power field (HPF) per length of the smear).

In present study, radiological presentations were divided in to eleven patterns which were not previously published in any literature as radiological pattern (RP) one (thick walled cavity with ragged internal margin), RP two (cavity with air fluid level), RP three (cavity with significant pericavitary consolidation), RP four (cavity/ /consolidation lesions merging with the hilum), RP five predominantly unilateral lesion), RP six (Predominantly lower lung field lesion), RP seven (extensive bilateral lesion without cavity), RP eight (pneumothorax/hydropneumothorax), RP nine (bilateral lesions any extent with any size cavity with upper and middle zone prepondrance), RP ten (unilateral lesions any extent with any size cavity with upper and middle zone preponderance) and RP eleven (bilateral/unilateral lesions non extensive and no cavity). The investigator who did the radiological reporting was unaware of sputum status and comorbidities.

Data are presented as mean \pm standard deviation (SD) using IBM SPSS ver. 20. Normal variables were analyzed using Chi-Square test, and when necessary, Fisher's Exact test. All hypotheses were constructed two-tailed and $p<0.05$ was considered significant.

\section{Results}

In TBDM group, most of the patients [39 (70.90\%)] were from the 40-59 years of age group whereas in TB group, most of the patients were in the 20-39 year age group (73.4\%) (Tab. 1). In present study duration of T2DM in all patients who were previously diagnosed ranged from 6 month to 10 years with mean of 4.21 \pm 1.86 years

Most of the patients in the TBDM group had high grade of positivity as compared to TB group. Six (12\%) and $23(41.8 \%)$ patients were having $3+$ grade in TB and TBDM groups respectively. Grade $2+$ was present in $11(22 \%)$ and $20(36.4 \%)$ patients, grade $1+$ was present in $15(30 \%)$ and $10(18.1 \%)$ patients and scanty was present in $18(36 \%)$ and $2(3.63 \%)$ patients in group TB and TBDM respectively $(p=0.0014)$.

\section{Discussion}

T2DM patients are very susceptible to tuberculosis infection because of weak immune system and have 2-3 times more risk for tuberculosis compared to people without T2DM [5].

Cavities are formed because of different pathological processes taking place in the lung. Presence of different type of pulmonary cavity can assist the clinician 
Table 1. Comparison of different parameters between both the groups

\begin{tabular}{|c|c|c|c|c|}
\hline Parameters & & TB & TBDM & P value \\
\hline Age (years)* & & $51.2 \pm 8.05$ & $39.5 \pm 9.2$ & 0.001 \\
\hline \multirow[t]{2}{*}{ Gender } & Male & $43(78.1)$ & $43(78.1)$ & NS \\
\hline & Female & $12(21.9)$ & $12(21.9)$ & NS \\
\hline \multirow[t]{2}{*}{ Residence } & Urban & $28(56)$ & $33(60)$ & NS \\
\hline & Rural & $22(44)$ & $22(40)$ & NS \\
\hline \multirow[t]{4}{*}{ Occupation } & WDFE & $11(22)$ & $18(32.7)$ & $<0.05$ \\
\hline & Farmer & $3(6)$ & $7(12.7)$ & NS \\
\hline & Household & $18(36)$ & $16(29.1)$ & NS \\
\hline & WTC & $10(20)$ & $5(9.1)$ & $<0.05$ \\
\hline \multirow[t]{5}{*}{ Symptomatology } & Cough & $33(66)$ & $45(81.8)$ & NS \\
\hline & Fever & $29(58)$ & $40(72.7)$ & NS \\
\hline & Haemoptysis & $5(10)$ & $22(40)$ & $<0.002$ \\
\hline & Breathlessness & $14(28)$ & $22(40)$ & NS \\
\hline & Weight loss & $23(46)$ & $11(20)$ & 0.0001 \\
\hline \multirow[t]{3}{*}{ Previous Contact } & Yes & $18(36)$ & $21(38.1)$ & NS \\
\hline & No & $22(44)$ & $25(45.45)$ & NS \\
\hline & ССТВ & $14(28)$ & $14(25.4)$ & NS \\
\hline \multirow[t]{2}{*}{ Comorbidities } & OAD & $2(4)$ & $5(9.1)$ & NS \\
\hline & Hypertension & $3(6)$ & $8(14.5)$ & NS \\
\hline \multirow[t]{3}{*}{ Addiction } & Smoker & $22(36.6)$ & $38(63.4)$ & NS \\
\hline & Alcoholic & $4(44.5)$ & $5(55.5)$ & NS \\
\hline & No history & $24(66.7)$ & $12(33.3)$ & NS \\
\hline \multirow[t]{4}{*}{ General examination } & Pallor & $18(36)$ & $12(21.8)$ & $<0.04$ \\
\hline & Icterus & $4(8)$ & $0(0)$ & NS \\
\hline & Clubbing & $10(20)$ & $6(10.1)$ & NS \\
\hline & Edema & $6(12)$ & $4(7.3)$ & NS \\
\hline
\end{tabular}

Data is expressed as no of patients (\%); ${ }^{*}$ data is expressed as mean \pm SD; WDFE - workers with occupational dust/fumes exposure; WTC — workers involving travel and crowded places; СCTB - close contact with known sputum positive pulmonary tuberculosis; OAD — obstructive airways disease; TBDM — tuberculosis with diabetes mellitus; TB - tuberculosis without diabetes mellitus; $p$ value $<0.05$ is considered as significant

in proper diagnostic evaluation. In infectious diseases such as mycobacterium tuberculosis, a cavity is the result of host and pathogen interaction. Identification of these cavities using radiological examination will assist the clinicians to evaluate the patients properly who are presented with pulmonary cavities [6].

In present study, we have identified 11 different pattern of radiological analysis, which revealed that pattern one, four and six were significantly different between both the groups. This was the unique analysis made in present study, which was not previously published. However, this should not be treated as universal classification for general use.

A study done by Alisjhabana et al that prevalence of T2DM as $14.8 \%$ with tuberculosis and was associated with older age and a greater body weight [7]. Patel et al studied 4,349 patients with tuberculosis infection and T2DM and reported the incidence rate of $5.77 \%$. The commonest age group was $40-60$ years
[8]. Present study is consistent with the Alisjhabana et al and Patel et al.

In present study, patients in TBDM group (51.2 \pm 8.05 years) were older than the patients in TB group (39.5 \pm 9.2 years). There were more males in TBDM group (78.1\% vs. $21.9 \%$ ) as compared to TB group. Mean age of patients reported by Patel A et al was also $50.5 \pm 13.5$ years which is similar to what is reported in present study [9].

Babu et al reported fever (93\%) as most common symptom in their study, followed by cough (45\%), weight loss (32\%), sputum production (29\%) and night sweets (15\%) [10]. But Bacakoglu et al reported that there were no effect of presence of T2DM on patients' symptomatology, bacteriology results, tuberculin reaction and localization of pulmonary infiltrates [11]. In our study however, hemoptysis was present in $40 \%$ patients in TBDM group which was significantly more than only $10 \%$ in TB $(p<0.002)$. Weight loss was present 
Table 2. Comparison of radiological findings between both the groups

\begin{tabular}{lccc}
\hline Radiological pattern & TB & TBDM & P value \\
\hline RP-1 & $2(4.4)$ & $12(15)$ & 0.01 \\
RP-2 & $2(4.4)$ & $4(6.6)$ & NS \\
RP-3 & $3(6.6)$ & $10(16.6)$ & NS \\
RP-4 & $02(4.4)$ & $10(16.6)$ & $<0.05$ \\
RP-5 & $06(13.3)$ & $16(26.6)$ & NS \\
RP-6 & $5(11.2)$ & $24(40)$ & $<0.0001$ \\
RP-7 & $07(4.4)$ & $15(25)$ & NS \\
RP-8 & $3(6.6)$ & $3(5)$ & NS \\
RP-9 & $10(22.3)$ & $5(8.3)$ & NS \\
RP-10 & $13(28.9)$ & $5(8.3)$ & NS \\
RP-11 & $18(40)$ & $10(16.6)$ & NS \\
\hline
\end{tabular}

Data is expressed as no of patients (\%); TBDM - tuberculosis with diabetes mellitus; TB - tuberculosis without diabetes mellitus; RP — radiological pattern; NS - not significant; pattern one - thick walled cavity with ragged internal margin; pattern two - cavity with air fluid level; pattern three - cavity with significant pericavitory consolidation; pattern four - cavity/consolidation lesions merging with the hilum; pattern five - predominantly unilateral lesion); pattern six - predominantly lower lung field lesion; pattern seven - extensive bilateral lesion without cavity; pattern eight — pneumothorax/hydropneumothorax; pattern nine - bilateral lesions any extent with any size cavity with upper and middle zone preponderance; pattern ten - unilateral lesions any extent with any size cavity with upper and middle zone preponderance; pattern eleven - bilateral/unilateral lesions non extensive and no cavity

in $20 \%$ of patients in TBDM group while in TB group it was seen in $46 \%$ patients. However, lack of weight loss in large number of patients in TBDM group compared to TB group was statistically significant $(p=0.0001)$. Other symptoms like cough, fever and breathlessness were similar in both the groups.

In our study, duration of T2DM ranged from 6 month to 10 years, with mean of $4.21 \pm 1.86$ years. Babu et al reported that duration of DM ranged from 2 years to 15 years, with mean $5.57 \pm 2.86$ years. The duration of DM in the cases positive for pulmonary tuberculosis ranged from 10 to 15 years with mean duration of $12.41 \pm 2.15$ years which is longer as compared to present study [10].

Bukhary et al reported that patients with TBDM had a higher bacillary load in sputum, more frequently positive culture at two month after anti-tubercular treatment and high rates of multi-drug resistant $M y$ cobacterium, presented with atypical radiographic features and T2DM may adversely affect the outcome of tuberculosis treatment [12].

Dooley et al concluded that T2DM alters immunity in tuberculosis patient, leading to higher baseline mycobacterial burdens and took longer times to culture conversion with treatment; a higher rate of relapse might result [13]. Three small retrospective studies suggested that baseline mycobacterial burdens might be higher in T2DM patients compared to control [14]. We also noticed that most of the T2DM patients in TBDM group had high grade of positivity as compared to controls.

James et al performed three studies and compared measures of tuberculosis severity at clinical presentation (including lung cavitary disease, sputum smear grade, and hemoptysis) in patients with and without T2DM. Their Study demonstrated that patients with tuberculosis and T2DM were more likely to present with higher sputum smear grade [adjusted odds ratio 2.63, 95\% confidence interval $(\mathrm{Cl}) 1.14-6.06]$ compared to tuberculosis patients without T2DM [15].

Sosman et al reported that a large proportion of T2DM patients with tuberculosis had lower-lung involvement, whereas non-diabetic patients usually had upper-lobe infiltrates. We also found that TBDM group patient had significantly more predominantly lower lung field lesions (40\%) as compared to TB group $(11.2 \%)$ ( $p=0.0003)$. It was widely believed that pulmonary tuberculosis in T2DM patient presented with an atypical radiographic pattern and distribution, particularly involving lower-lung [16].

Carreira et al reviewed the records of 123 patients with tuberculosis and T2DM and compared with another 123 patients with tuberculosis without T2DM. In both groups multi-lobar lung lesions predominated, there were more cases of isolated lower lung field involvement in diabetics than in non-diabetics (10.6\% vs. $3.3 \%, p=0.03)$. Cavitary lesions were less frequent (63.4\% vs. $82.1 \%, p=0.01)$ [17]. In our study cavitary lesion was common presentation in T2DM patients. Thick walled cavity with ragged internal margin was present in 15\% patient in TBDM group as compared to $4.4 \%$ in TB group ( $p=0.01)$.

Other presentations noticed in T2DM patients were cavity or consolidation lesions merging with the hilum, it was found more often in T2DM patients (16.6\%) as compared to patients without T2DM (4.4\%) $(p=0.04)$. Other findings which were also common in T2DM patients like cavity with air fluid level and pericavitary consolidation were present more often in T2Dm patients $(\mathrm{p}>0.05)$.

The present study has limitation, namely small sample size and not done building; a large randomized blinded clinical trial is required to confirm the results. Age of patients in both the groups was not comparable, which may have impact on the results. Since it was a prospective study, follow up of patients was not done, which would have been beneficial for the present study. 


\section{Conclusion}

Present study has found major differences in clinical and radiological presentations in patients of tuberculosis and T2DM as compared to patients without T2DM.

\section{REFERENCES}

1. Bailey SL, Grant P. The tubercular diabetic: the impact of diabetes mellitus on tuberculosis and its threat to global tuberculosis control. Clin Med 2011; 11: 344-347.

2. Chaya BE, Vishwakumar SN. A study of pulmonary tuberculosis in diabetes mellitus and its clinicoradiological correlation. Indian Journal of Basic and Applied Medical Research 2015; 4: 30-38.

3. IDF Diabetes atlas, $7^{\text {th }}$ edition, 2015.

4. Bashar M, Alcabes P, Rom WN, Condos R. Increased incidence of multidrug-resistant tuberculosis in diabetic patients on the Bellevue Chest Service, 1987 to 1997. Chest 2001; 120: 1514-1519.

5. Syed Sulaiman SA, Mohd Zain FA, Abdul Majid S, Munyin N, Mohd Tajuddin NS, Khairuddin Z et al. Tuberculosis Among Diabetic Patient. Web med Central Infectious Diseases 2011; 2: 1-13.

6. Gadkowski LB, Stout JE. Cavitary Pulmonary Disease. Clin Microbiol Rev 2008; 21: 305-333.

7. Alisjahbana B, Sahiratmadja E, Nelwan EJ. The effect of type 2 diabetes mellitus on the presentation and treatment response of pulmonary tuberculosis. Clin Infect Dis 2007; 45: 428-435.
8. Patel JC, Cheryl SD, Jigjini SS. Diabetes and Tuberculosis. Ind J Tub 1977; 24: 155-158.

9. Patel AK, Kiran R, Feroz G. Clinical Profile of Sputum Positive Pulmonary Tuberculosis Patient With Diabetes Mellites. National Journal of Medical Research 2012; 2: 1-6.

10. Babu RV, Manju R, Kumar SV, Das AK. A Comparative Study of Diabetes Mellitus in Pulmonary Tuberculosis Patients. World Journal of Medical Sciences 2013; 9: 93-96.

11. Bacakoglu F, Basoglu OK, Cok G, Sayiner A, Ates M. Pulmonary tuberculosis in patients with diabetes mellitus. Respiration 2001; 68: 595-600.

12. Bukhary ZA. Rediscovering the Association between Tuberculosis and Diabetes Mellitus: A Perspective. JTU Med Sc 2008; 3: 1.

13. Dooley KE, Chaisson RE. Tuberculosis and diabetes mellitus: convergence of two epidemics. Lancet Infect Dis 2009; 9: 737-746.

14. Wang CS, Yang CJ, Chen HC. Impact of type 2 diabetes on manifestations and treatment outcome of pulmonary tuberculosis. Epidemiol Infect 2009; 137: 203-210.

15. James MM. Diabetes mellitus and active tuberculosis disease: Clinical presentation and treatment outcomes in adult tuberculosis patients. 2013. Accessed on 4 Feb 2016. http://pid.emory. edu/ark:/25593/f4cng.

16. Sosman MC, Steidl JH. Diabetic tuberculosis. Amer J Roentgenol 1927; 17: 625-629.

17. Carreira S, Costeira J, Gomes J, André JM, Diogo N. Impact of diabetes on the presenting features of tuberculosis in hospitalized patients. Rev Port Pneumol 2012; 18: 239-243. 